\title{
La situación de E/LE en las universidades chinas y la aplicación de doblaje en la enseñanza para los cursos superiores
}

MENGZHI LIAN

Universidad Politécnica de Valencia

menlia@doctor.upv.es

\begin{abstract}
Resumen: En la actualidad, en la enseñanza universitaria de español como lengua extranjera del tercer y cuarto curso, parece que la organización de los cursos y el método tradicional en la enseñanza no pueden satisfacer las necesidades de los alumnos, por eso sería necesario introducir métodos nuevos en la enseñanza. En este trabajo, en primer lugar, presentaremos brevemente las habilidades que más necesitan desarrollar los alumnos del periodo superior; en segundo lugar, se analizarán las posibilidades y ventajas de introducir el doblaje en la enseñanza; por lo último, se presentarán unos modelos básicos y unos principios de este método didáctico.
\end{abstract}

Palabras clave: estudiantes chinos, periodo superior, demandas, doblaje

The situation of E/LE in Chinese universities and the application of dubbing in teaching for higher education

Abstract: For Chinese students of Hispanic philology of the upper period, they have to face some challenges, however, the organization of courses and the traditional method of teaching cannot satisfy their needs well, so it would be necessary to introduce new methods in the classroom of this period. In this article, first, we will briefly introduce the situation of E/LE in Chinese universities and analyze what are the skills that need to be more developed by the students of the superior period; Second, we discuss the possibilities and advantages of introducing dubbing into teaching; for the last, the models and the principles of this didactic method will be presented.

Key words: Chinese students, higher period, demands, dubbing

\section{Introducción}

En este artículo intentamos introducir el doblaje en la enseñanza de español como lengua extranjera en los periodos superiores (tercer y cuarto curso) de las facultades de filología hispánica en las universidades chinas. En esta etapa de la enseñanza, el fortalecimiento de las habilidades y los conocimientos básicos lingüísticos todavía ocupan un puesto predominante, y no se presta atención suficiente a la práctica de las destrezas en contextos reales.

En el doblaje se compromete la comprensión auditiva, la transferencia lingüística en contextos diversos, y también exige la competencia transcultural. Por eso, en este trabajo intentamos discutir sobre la posibilidad de introducir esta actividad como método didáctico en la enseñanza diaria de este periodo. 
En este trabajo, en primer lugar, se presentará de manera muy breve los problemas de E/LE en las universidades de China en el periodo superior, y se verán las necesidades más importantes y pertinentes de los alumnos; en segundo lugar, se explicarán las ventajas de la aplicación de doblaje en la enseñanza, y también unos modelos básicos (en el contexto de la E/LE de china) de realizar estas actividades; por último, se analizarán unos principios que pueden ser importantes en la práctica de este método.

\section{Las necesidades de los alumnos chinos universitarios en los cursos tercero y cuarto}

Para los alumnos chinos de filología hispánica del tercer y cuarto curso, los desafíos son más diversos: además de seguir profundizando sus conocimientos lingüísticos del español y fomentando las destrezas básicas de este idioma, tienen que saber cómo usar esta lengua en su futuro trabajo. En este apartado, a través del análisis sobre los resultados de EEE 8 y la situación de empleo del alumnado de la facultad de filología hispánica, intentamos saber cuáles son las habilidades más importantes para los alumnos chinos de este periodo.

Consultado los resultados del EEE8 durante los años 2005 y 2013, se observa que los alumnos manifiestan dificultades notables en las siguientes partes (Chang y Liu 2015:199-212)

\section{La elección de vocablos}

2. La explicación con español las expresiones especiales

3. Comprensión auditiva

\section{Interpretación (sight-translation)}

En la primera y segunda parte se evalúan unas técnicas retóricas o expresiones coloquiales, y se evalúan las competencias sociolingüísticas de los alumnos

En cuanto a la situación del empleo, según una encuesta hecha a los alumnos de 40 facultades de español del año 2013 (Wang y Zheng 2015: 290), la mayoría de los alumnos (66.5\% de total) del cuarto curso se inclinan por trabajar en empresas de comercio después de graduarse.

Según las opiniones de las empresas, los alumnos tienen que fomentar más su expresión y comprensión oral y su interpretación. Las opiniones de los alumnos graduados nos revelan que en el trabajo también es muy importante la competencia intercultural y los conocimientos de español pragmático comercial (Cui 2015: 117).

Basándonos en los análisis de arriba, podríamos sacar la conclusión de que: para los alumnos chinos de filología hispánica de tercer y cuarto curso, las siguientes habilidades o competencias serían las más pertinentes:

1. Entre las destrezas básicas: la comprensión y expresión auditiva

2. Entre las competencias: la competencia sociolingüística y la competencia intercultural

3. Las habilidades de aplicación del idioma: la interpretación (sight-translation) 
En el siguiente apartado, exploraremos la posibilidad de fomentar estas habilidades y competencias con las actividades de doblaje.

\section{La aplicación del doblaje en la E/LE}

La aplicación del doblaje en la enseñanza de lengua extranjera ya fue mencionada en Duff (1989). En este trabajo, por la primera vez el doblaje fue incluido entre las medidas didácticas en el aula de LE. Después, se publicaron sucesivamente varios trabajos en que se discuten las ventajas, los modelos y posibilidades del uso del doblaje en la enseñanza de lengua extranjera, como los de Kumai (1996), Burston (2005), Wagener (2006), etc. Hay artículos que tratan de la aplicación real del doblaje en la enseñanza (Danan 2010). Durante el año 2010 y el 2015, salen varias investigaciones importantes en la investigación de esta orientación, como la de Chiu (2012), Navarrete (2013) y de Talaván, de Ávila-Cabrera (2015) y de Sánchez Requena (2015). Estos trabajos nos demuestran el valor pedagógico del doblaje en la enseñanza, y nos da toda la razón para aplicarlo en la E/LE en el contexto chino.

\subsection{Las ventajas de la aplicación de doblaje en $E / L E$ en el periodo superior}

En el contexto chino, en muchas universidades chinas, se celebran concursos de doblaje de las películas de lengua extranjera entre los estudiantes. No obstante, en estos casos, el doblaje es considerado una medida para provocar el interés por el aprendizaje más que un método didáctico, sin embargo, esto no es la única función de este método. Se enumerarán las ventajas de uso didáctico de doblaje en el aula de E/LE, y se analizará sí sus ventajas pueden ayudar a fomentar las habilidades y competencia a las que aludimos en el apartado anterior.

\section{El fomento de habilidades distintas}

El doblaje pude mejorar diversas destrezas, sobre todo las habilidades orales que todavía deben cobrar más atención en la enseñanza de LE en China. Durante la actividad, los alumnos van a imitar la manera de hablar de los actores, como indica Burston (2005: 80). para mantener la sincronización, los alumnos van a imitar la velocidad de habla de los personajes, que también es la nativa. Es decir, esta actividad no sólo permite a los alumnos escuchar y hablar, sino también que les ofrece un español más auténtico y vivo que encuentran en sus manuales para aprender e imitar. Además, según el diseño de las actividades y los materiales que elegimos, se puede desarrollar otras habilidades como la de interpretación, traducción y usos de español en contextos determinados, esto se verá con más detalles en el siguiente apartado.

2. La introducción de nuevos métodos didácticos en la E/LE de china.

En el E/LE de China todavía domina el enfoque tradicional de gramáticatraducción. Es difícil irrealista cambiar este sistema a corto plazo. No obstante, la aplicación de métodos nuevos puede recompensar las desventajas de los métodos tradicionales.

\section{Provocar el interés por el aprendizaje}

Según Santos Rovira, debido a la tradición educativa china, como en muchas otras disciplinas, según el concepto de muchas facultades de filología hispánica, parece 
que el dominio de un idioma siempre «implica un gran esfuerzo por parte de los alumnos», y se suele aprender conocimientos a través de «la memorización y repetición de contenidos y actividades» (2011: 64). No sería difícil imaginar que esto hace que el aprendizaje del español se convierta en una actividad aburrida, dolorosa y tediosa, y daña en cierta medida la motivación de los alumnos. La introducción del doblaje en el aula de E/LE puede hacer más divertido el aprendizaje. En comparación con los textos en los manuales, el material audiovisual provoca el interés de los alumnos con más facilidad, aparte de esto, con diferentes diseños del procedimiento, se puede gamificar las actividades didácticas.

\section{Los modelos básicos de actividades de doblaje}

Según la necesidad real, las actividades de doblaje con objetivo didáctico pueden contar con grandes diversidades. En este apartado, nos limitamos a presentar sólo unos de los modelos más básicos.

Según Chaume (2012: 29-31), generalmente los procedimientos del doblaje comercial consisten en:

1. Elegir la película y contactar con el estudio de doblaje

2. Contactar con un traductor y organizar el procedimiento.

3. Producir un esbozo de traducción

4. Adaptación del esbozo

5. Seleccionar los actores de doblaje

6. Doblar

Para su aplicación en la enseñanza, podemos reducir estos procesos en cuatro principales: 1) la selección de material; 2) la obtención del texto; 3) la transferencia lingüística; 4) la producción (el doblaje). Además, también es importante atender el idioma del material.

A continuación, se presentan los modelos más básicos de las actividades de doblaje según el idioma del material a trabajar.

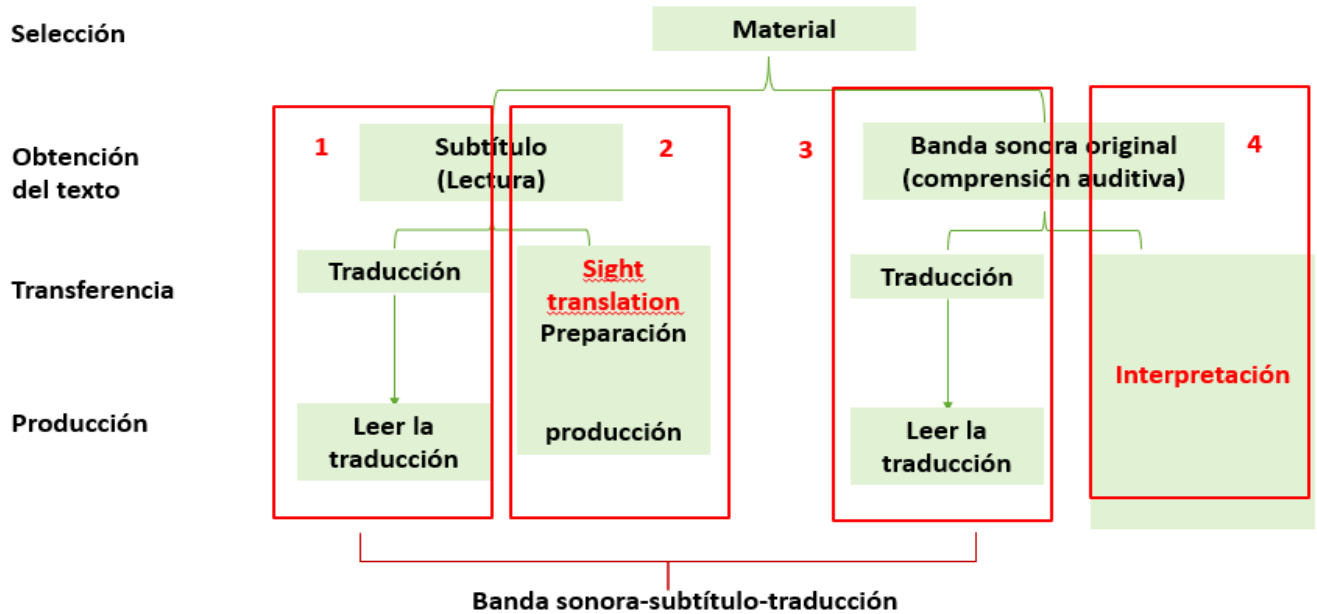


Imagen 1. Modelos básicos de las actividades de doblaje con material de idioma español

Con los materiales de lengua española, tenemos principalmente cuadro modelos básicos de actividades. Cabe mencionar que, según la manera (con o sin subtítulo, con lectura o comprensión oral) de obtener el contenido del material, el proceso y la dificultad de la actividad pueden ser diferente.

1. Leer y traducir el subtítulo y doblar la traducción

2. Leer el subtítulo y prepararse para una interpretación (sight-translation)

3. Escuchar y comprender el contenido del material, traducirlo y doblarlo

4. Escuchar el material e interpretarlo

Para los alumnos chinos universitarios de español, que todavía necesitan mejorar sus habilidades orales, los modelos que contiene actividad de comprensión y expresión auditiva significaría mayor dificultad para ellos. Es decir, el modelo uno sería el más fácil mientras el cuatro sería el más difícil.

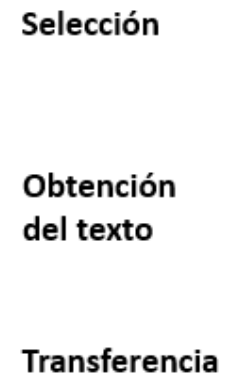

Producción

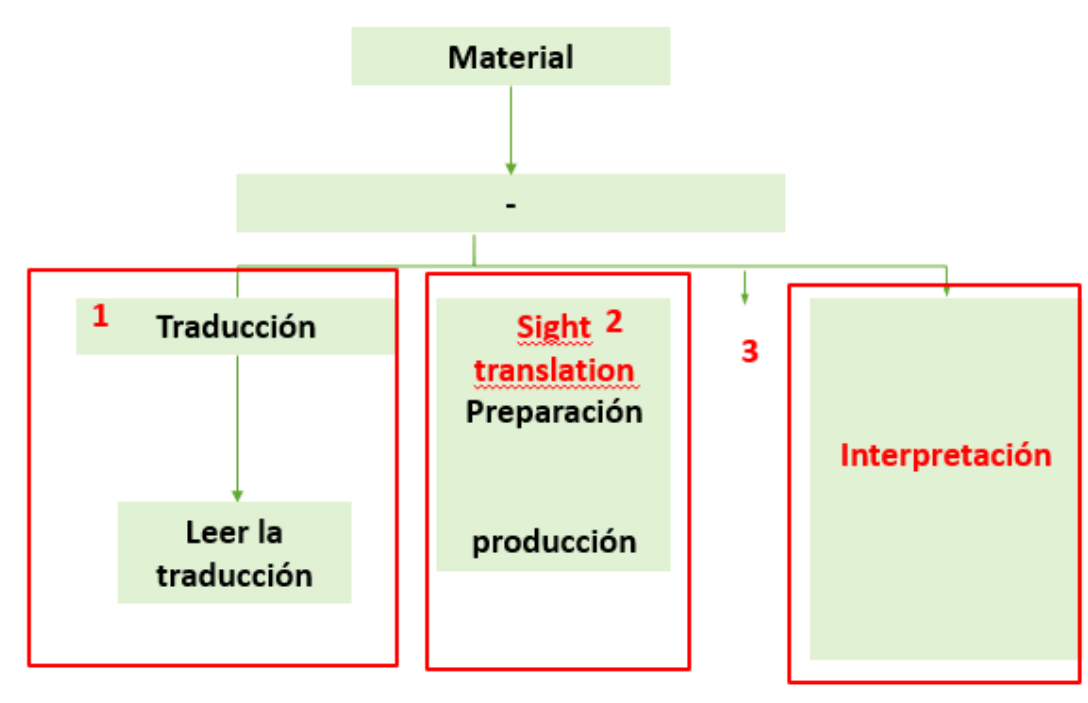

Imagen 2. Modelos básicos de las actividades de doblaje con material de idioma chino (la lengua materna)

Si trabajamos con los materiales de chino, los modelos son más simples, como el idioma original es el materno, no hay diferencia en la fase de obtención del texto. En este caso, tenemos tres modelos principales de actividad:

1. Comprender el texto original, traducirlo y doblarlo

2. Comprender el texto original y realizar una "sight-translation" después de unos minutos de preparación

3. Comprender (escuchar) el texto original e interpretarlo

Cuando trabajamos con material de este carácter, la dificultad de cada modelo varía según la manera de transferir el idioma y la de producir el texto traducido. Para los alumnos chinos, el tercer modelo sería más difícil y el más fácil sería el primero. 
En este apartado, se presentan los modelos más básicos que se pueden usar en las actividades de doblaje como medida didáctica. En la aplicación real, podemos diversificarlos con actividades complementarias.

\section{Unos principios en la aplicación de E/LE para los alumnos chinos}

En este apartado, se presentan unos aspectos imprescindibles en la aplicación del doblaje en la enseñanza de E/LE.

\subsection{Diseñar y adaptar las actividades según la necesidad de los alumnos}

Para que este método pueda ayudar a desarrollar las habilidades que quieren o necesitan fomentar los alumnos, en primer lugar, es importante saber cuáles son sus necesidades.

Los estudiantes podrían manifestar su interés por:

1. Desarrollar ciertas destrezas básicas: compresión y comprensión escrita o auditiva

2. Fomentar la aplicación del idioma: interpretación, traducción, interpretación

3. Obtener conocimientos del español en diferentes ocasiones: usos coloquiales, español pragmático comercial, entre muchos otros

4. Los conocimientos culturales de países hispanohablantes.

Podemos adaptar nuestra actividad a estas necesidades con diferentes medidas:

En primer lugar, se puede elegir el modelo básico de las actividades según nuestro objetivo didáctico. En el apartado 4, se pudo ver que los modelos básicos abarcan la formación de habilidades diversas, y cada uno de ellos enfatiza determinadas habilidades, podemos elegir uno según la necesidad

En segundo lugar, se puede elegir el material de trabajo según el tema, y el estilo de dialogo. Los materiales a trabajar pueden ser películas, telenovelas, anuncios, entre muchos otros, tienen diferentes temas y características lingüísticas. Podemos elegir el material según el interés y la necesidad de los alumnos.

También se puede complementar las actividades con otras actividades. Si con los modelos básicos y los materiales todavía no se puede satisfacer las demandas didácticas, sería una buena opción complementarlos con otras actividades. Por ejemplo, para practicar la destreza de redacción, podemos pedir que los alumnos escriban la continuación de la película. Para fomentar su expresión oral, podemos hacer concursos pequeños de debate sobre un tema determinado. Estas actividades son una extensión del doblaje y pueden diversificar bastante el contenido en la clase.

\subsection{Ajustar la dificultad de las actividades según el nivel lingüístico de los alumnos}

La dificultad de la actividad puede influir en la motivación de participación de los alumnos. Por un lado, si el nivel de las actividades es demasiado superior al de los alumnos, se pueden desmotivar; por otro lado, si es demasiado fácil para ellos, la función de fomento de las actividades será limitada, y la falta de desafío también podría 
aburrir a los alumnos. Por eso, sería recomendable que los profesores supieran claramente el nivel, que tienen los estudiantes en las habilidades orales.

Podemos controlar la dificultad aplicando diferentes modelos de actividad. Como se ha analizado en los apartados anteriores, los modelos básicos que exigen más habilidades orales (como la comprensión auditiva, la interpretación) significan más dificultad para los alumnos chinos.

Sin embargo, a veces, con el objetivo didáctico fijados, sería difícil cambiar el modelo. En este caso, podemos jugar con la dificultad de los materiales audiovisual a trabajar.

La dificultad de comprender de material audiovisual puede varía según la procedencia del material. Generalmente, los materiales chinos causarán menos presión a los alumnos; y entre los materiales españoles, los de América Latina serían más difíciles de entender. Porque muchos profesores chinos de español toman el español peninsular como estándar, y presentan poco el español latinoamericano, esto causa que éste último cause mayor dificultad de comprensión para los alumnos chinos.

El tema a veces también influiría en la dificultad del material. Para entender los materiales de ciertos temas, serían necesarios unos conocimientos de fondo - como los culturales- o términos relativos - como los de economía, de tecnología-, y sería conveniente una previa preparación.

A lo mejor, uno de los factores que afectaría la dificultad de materiales a trabajar consiste en las características lingüísticas del material, como la velocidad de habla, el nivel del vocabulario, la fluencia, el acento, etc. (Richard 1983: 234).

Si bien existirían otros métodos para controlar la dificultad de las actividades de doblaje, en este artículo nos limitamos a presentar sólo unos de ellos. Si queremos utilizar el doblaje a largo plazo en nuestra enseñanza, sería recomendable empezar con un nivel básico, y elevar gradualmente su dificultad según el progreso de los alumnos.

\section{Conclusión}

En este artículo, hemos visto las posibilidades, los modelos básicos y unos principios de la aplicación didáctica del doblaje enseñanza del español como lengua extranjera, sobre todo en la E/LE de las universidades chinas para los alumnos del tercer y cuarto curso.

Se han analizado, en primer lugar, las habilidades y competencias que necesitan fomentar los alumnos chinos de filología hispánica en el tercer y cuarto curso. En este periodo, es importante desarrollar las habilidades orales, habilidades como interpretación, sus competencias sociolingüísticas e interculturales.

Para mejorar la calidad de la enseñanza de este periodo, y satisfacer las necesidades reales de los alumnos, creemos que el doblaje puede ser una medida adecuada. Porque, en primer lugar, como método didáctico, puede desarrollar diferentes destrezas y competencias de los alumnos, sobre todo las cuales que no reciben atención suficiente en la enseñanza con una metodología tradicional, como las habilidades orales y la competencia sociolingüística, e incluso podemos diversificar este método con actividades complementarias. En segundo lugar, como un método todavía algo novedoso para la enseñanza de lengua extranjera de China, se pueden introducir nuevos conceptos e ideas en la enseñanza de español. El doblaje no resolverá a corto plazo 
todos los problemas, ayudaría a superar unos límites del sistema actual; en tercer lugar, puede motivar el aprendizaje de los alumnos.

A continuación, enumeramos los modelos básicos de las actividades de doblaje que se pueden realizar. Según sus contenidos, y teniendo en cuenta las características de aprendizaje de los alumnos chinos en este periodo, cada modelo tiene niveles diferentes de dificultad. Se puede ver que, en estos modelos, parece que la comprensión auditiva, la interpretación (incluye el sight-translation), y la traducción son las habilidades más practicadas. Cabe mencionar que, si queremos desarrollar otras habilidades, podemos complementar estos modelos con otras actividades, y esto depende de la creación del profesor (o de los alumnos también).

Para que este método funcione mejor, al diseñar las clases, hay que tener en cuenta unos principios. En este trabajo, se han presentado los dos que según nuestros criterios son más importantes. El primero trata de adaptar las actividades según las demandas didácticas, con el cambio de modelos, del material a trabajar y con las actividades complementarias. El segundo principio consiste en controlar la dificultad de las actividades en un nivel adecuado para no desmotivar a los alumnos, se puede realizar con optar por seguir diferentes modelos, y también es una buena opción equilibrar la dificultad de la actividad con materiales más fácil de comprender.

En resumen, como un método didáctico, el doblaje puede ayudar a desarrollar las habilidades y competencias más pertinentes para los alumnos chinos en el tercer y cuarto curso de la especialidad de filología hispánica. Todo apunta a que, en el futuro no muy lejano, este método será más aceptado y aportará más posibilidades a la E/LE en China.

\section{Bibliografía}

DANAN, Martine (2010). «Dubbing projects for the language learner: A framework for integrating audiovisual translation into task-based instruction». Computer Assisted Language Learning, 23, 5, 441-456.

BURSTON, Jack (2005). «Video dubbing projects in the foreign language curriculum». CALICO Journal, 23, 1, 72-79.

CHANG, Fu-liang, y LIU, Yuan-qi (2015). «La investigación sobre las situaciones de EEE 8 en las facultades de filología hispánica en los institutos superiores en China». En S. Zheng, (ed.); La investigación sobre las enseñanzas de español en los institutos superiores de China. (pp. 193-280) Pekín: Editorial de enseñanza e investigación de lenguas extranjeras.

CuI, Qing-xia (2015). «La primera exploración sobre la situación actual de las organizaciones de los cursos de Filología Hispánica en el grado». En El diario académico del Instituto de Tecnología de Chifeng, 12, 117-119.

Chaume, Federica (2012). Audiovisual translation: Dubbing. Manchester: St. Jerome Publishing.

CHIU, Yi-hui (2012). "Can film-dubbing projects facilitate EFL learners' acquisition of English pronunciation? ». British Journal of Educational Technology, 43, 1, 24-27. 
Duff, Alan (1989). Translation. Oxford: Oxford University Press.

GRUPO DE ElABORACIÓN DEL Plan CURRICULAR DE ESPAÑOL PARA LICENCIATURA EN Filología HispániCA (2000). Plan Curricular de Español para Licenciatura en Filología Hispánica. Shanghai: Editorial de la enseñanza de lenguas extranjeras de Shanghai.

KUMAI, William Naoki (1996). «Karaoke movies: Dubbing movies for pronunciation». The Language Teacher Online, 20, 9.

NAVARRETE, Marga (2013). «El doblaje como herramienta en el aula de español y desde el entorno ClipFlair». MarcoELE, 16, 75-87.

QUEROL BATALler, María (2014). «La especialidad de español como carrera universitaria en China». En Marco ELE: Revista Didáctica 18, 1-12.

RiCHARD, Jack C. (1983). «Litening Comprehension: Approach, Design, Procedure». TESOL Quarterly, 17, 60-81.

SÁNCHEZ-REQUENA, Alicia (2015). «Audiovisual Translation in Teaching Foreign Languages; Contributions of Dubbing to Develop Fluency and Pronunciation in Spontaneous Conversations». Porta Linguarum, 26, 9-21.

SANTOS Rovira, José María (2011). La enseñanza del español en China: historia, desarrollo y situación actual. Lugo: Editorial Axac.

TAlavÁn, Noa y Ávila-CABRERA, José Javiel. (2015). «First Insights into the combination of dubbing and subtitling as L2 didactic tools». En A. Caimi and C. Mariotti (eds.); Subtitles and Language Learning. (pp. 149-172). Oxford: Peter Lang.

WANG, Meng-meng y ZHENG, Shu-jiu (2015). «La investigación sobre las situaciones de los alumnos de la especialidad española en los institutos superiores». En S. Zheng, (ed.); La investigación sobre las enseñanzas de español en los institutos superiores de China. (pp. 281-259). Pekín: Editorial de enseñanza e investigación de lenguas extranjeras.

ZHENG, Shu-jiu (2015). «El prólogo: las oportunidades, desafíos y contramedidas de la ELE en los institutos superiores». En S. Zheng, (ed.); La investigación sobre las enseñanzas de español en los institutos superiores de China. (pp. 1-6). Pekín: Editorial de enseñanza e investigación de lenguas extranjeras. 